\title{
High-temperature nonequilibrium Bose condensation induced by a hot needle
}

\author{
Alexander Schnell, ${ }^{1, *}$ Daniel Vorberg, ${ }^{1}$ Roland Ketzmerick, ${ }^{1,2}$ and André Eckardt ${ }^{1, \dagger}$ \\ ${ }^{1}$ Max-Planck-Institut für Physik komplexer Systeme, Nöthnitzer Straße 38, 01187 Dresden, Germany \\ ${ }^{2}$ Technische Universität Dresden, Institut für Theoretische Physik and Center for Dynamics, 01062 Dresden, Germany
}

(Dated: July 28, 2017)

\begin{abstract}
We investigate theoretically a one-dimensional ideal Bose gas that is driven into a steady state far from equilibrium via the coupling to two heat baths: a global bath of temperature $T$ and a "hot needle", a bath of temperature $T_{h} \gg T$ with localized coupling to the system. Remarkably, this system features a crossover to finite-size Bose condensation at temperatures $T$ that are orders of magnitude larger than the equilibrium condensation temperature. This counterintuitive effect is explained by a suppression of long-wavelength excitations resulting from the competition between both baths. Moreover, for sufficiently large needle temperatures ground-state condensation is superseded by condensation into an excited state, which is favored by its weaker coupling to the hot needle. Our results suggest a general strategy for the preparation of quantum degenerate nonequilibrium steady states with unconventional properties and at large temperatures.
\end{abstract}

Introduction.-In thermal equilibrium the state of a system is strongly restricted by the laws of thermodynamics. Irrespective of the details of the environment, it is characterized by a few state variables only, like temperature or chemical potential. This is not the case anymore, when the system is driven far away from equilibrium and the exciting question arises, whether the freedom to prepare nonthermal states of matter can be used to manipulate the properties of many-body quantum systems in a controlled fashion. Recently, this led to various interesting directions of research. In transient states, dynamically induced Bose condensation [1,2], light-induced superconductivity [3], and dynamical phase transitions [4-6] were studied. Nonequilibrium steady states, whose properties depend on the initial condition, can occur in many-body localized isolated systems [7-11], including Floquet (i.e. time-periodically driven) systems [12, 13] such as discrete time crystals [14-18]. Floquet engineering, the coherent control of isolated systems by periodic driving on long (but finite) time scales, was very successfully used in atomic quantum gases, e.g. for the realization of artificial magnetic fields [19, 20]. Also nonequilibrium steady states of driven-dissipative manybody systems attracted considerable attention, including open Floquet systems [21-30] and photonic systems [31], where i.a. the question was studied in how far Bose condensation can be distinguished from lasing [32-34].

In this work we investigate the nonequilibrium steady state of a quantum gas in contact with two heat baths of different temperature. In particular, we consider a one-dimensional (1D) ideal Bose gas that is coupled globally to an environment of temperature $T$ and driven into a steady state far from equilibrium via the additional coupling to a "hot needle", a local bath of temperature $T_{h} \gg T$ [Fig. 1(a)]. We find the surprising effect that a crossover to Bose condensation can occur when both temperatures $T$ and $T_{h}$ are orders of magnitude larger than the temperature where (finite-size) condensation occurs in equilibrium. We explain this behavior by a suppression (a)
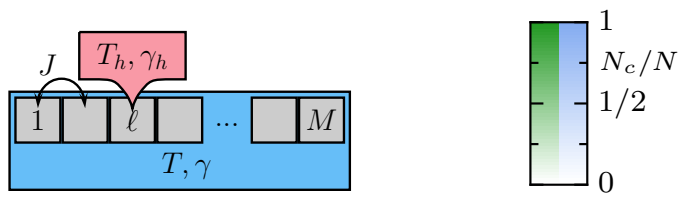

(b)

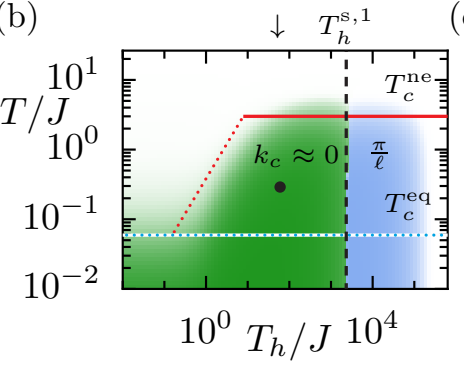

(c)

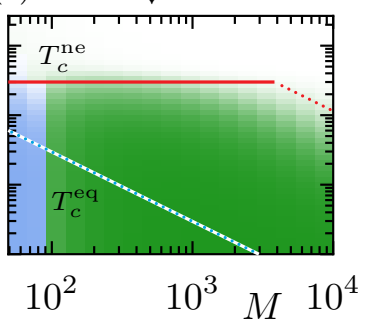

FIG. 1. (color online) (a) Tight-binding chain with $N=n M$ bosons on $M$ sites and tunneling parameter $J$, coupled with strengths $\gamma$ and $\gamma_{h}$ to a global bath of temperature $T$ and to a "hot needle" of temperature $T_{h}$ at site $\ell$, respectively. (b,c) Condensate fraction $N_{c} / N$ indicated by green (blue) shading for ground-state (excited-state) condensation in the mode $k_{c} \approx 0\left(k_{c} \approx \pi / \ell\right)$ versus $T$ and $T_{h}$ or $M$; for $\ell=5, \gamma_{h}=0.5 \gamma$, $n=3$ and (b) $M=500$ or (c) $T_{h}=60 J$. Estimated temperature $T_{c}^{\text {ne }}$ for 1D-like (3D-like) Bose condensation plotted as red dotted (solid) lines. Blue-white dotted lines give equilibrium condensation temperature $T_{c}^{\text {eq }}\left(\right.$ for $\gamma_{h}=0$ ). Black dashed line gives estimated needle temperature $T_{h}^{\mathrm{s}, 1}$, where excited-state condensation sets in. Black arrows indicate the parameters where (b) and (c) coincide.

of long-wavelength excitations resulting from the competition between both baths. Moreover, we observe that for sufficiently large needle temperatures Bose condensation occurs in an excited state of the system, which provides a better decoupling from the hot needle. This intriguing phenomenon bears resemblance to the quantum Zeno effect.

System and model.- - Let us consider a 1D system of $N$ noninteracting bosons that tunnel between adjacent sites of a tight-binding chain of length $M$ [Fig. 1(a)]. 
The Hamiltonian reads

$$
\hat{H}=-J \sum_{i=1}^{M-1}\left(\hat{a}_{i+1}^{\dagger} \hat{a}_{i}+\text { h.c. }\right)=\sum_{k} \varepsilon_{k} \hat{n}_{k} .
$$

Here $J$ is the tunneling parameter and $\hat{a}_{i}$ the bosonic annihilation operator at lattice site $i$. The dimensionless wave numbers $k=\pi \nu /(M+1)$ with $\nu=$ $1, \ldots, M$ characterize the single-particle energy eigenmodes with energy $\varepsilon_{k}=-2 J \cos (k)$, wave function $\langle i \mid k\rangle=\sqrt{2 /(M+1)} \sin (k i)$ (describing a superposition of states with quasimomenta $k$ and $-k$ ), and number operator $\hat{n}_{k}=\hat{c}_{k}^{\dagger} \hat{c}_{k}$ with $\hat{c}_{k}=\sum_{i}\langle k \mid i\rangle \hat{a}_{i}$. The eigenstates of the Hamiltonian are Fock states $|\boldsymbol{n}\rangle$ labeled by the vector $\boldsymbol{n}$ of occupation numbers $n_{k}$.

A heat bath $b$ is modeled as a collection of harmonic oscillators in thermal equilibrium with temperature $T_{b}$ that couple to a single-particle system operator $\hat{v}^{(b)} \equiv \sum_{q k} v_{q k}^{(b)} \hat{c}_{q}^{\dagger} \hat{c}_{k}$. In the limit of weak systembath coupling (small compared to $\min _{k \neq q}\left\{\left|\Delta_{q k}\right|\right\} \approx$ $1.5 \mathrm{~J} / M^{2}$, with $\Delta_{q k} \equiv \varepsilon_{q}-\varepsilon_{k}$ ), the bath induces quantum jumps between the energy eigenstates $|\boldsymbol{n}\rangle$ of the system, where a boson is transferred from mode $k$ to mode $q$ with rate $\left(n_{q}+1\right) n_{k} R_{q k}^{(b)}$. Here the dependence on the occupation $n_{q}$ reflects the bosonic quantum statistics. The single-particle rate $R_{q k}^{(b)}$ is obtained within the rotating-wave Born-Markov approximation and is given by the golden-rule-type expression $R_{q k}^{(b)}=$ $\frac{2 \pi}{\hbar}\left|v_{q k}^{(b)}\right|^{2} J_{b}\left(\Delta_{q k}\right)\left[\exp \left(\Delta_{q k} / k_{\mathrm{B}} T_{b}\right)-1\right]^{-1}[35]$. We choose ohmic baths with spectral density $J_{b}(\Delta) \propto \Delta$. Setting $\hbar=k_{\mathrm{B}}=1$ from now on, the rates take the form

$$
R_{q k}^{(b)}=f_{q k}^{(b)} \gamma_{b}^{2} \frac{\Delta_{q k}}{e^{\Delta_{q k} / T_{b}}-1} \stackrel{T_{b} \gg\left|\Delta_{q k}\right|}{\longrightarrow} f_{q k}^{(b)} \gamma_{b}^{2} T_{b},
$$

with the dimensionless coupling strength $\gamma_{b}$ and factor $f_{q k}^{(b)} \propto\left|v_{q k}^{(b)}\right|^{2}$. When deriving analytical estimates, the asymptotic expression for $T_{b} \gg\left|\Delta_{q k}\right|$ will be employed for the hot bath and for long-wavelength modes $k, q \ll 1$, otherwise the full expression is used. We also define the rate asymmetry

$$
A_{q k}^{(b)}=R_{q k}^{(b)}-R_{k q}^{(b)}=-f_{q k}^{(b)} \gamma_{b}^{2} \Delta_{q k} .
$$

We will consider two baths, $R_{q k}=R_{q k}^{(g)}+R_{q k}^{(h)}$, a global bath $g$ of temperature $T$ and coupling strength $\gamma$ as well as a hot local bath $h$ at site $\ell$ (the hot needle) of temperature $T_{h}$ and coupling strength $\gamma_{h}$ [Fig. 1(a)]. The hot needle couples to the operator $\hat{v}^{(h)}=\hat{a}_{\ell}^{\dagger} \hat{a}_{\ell}$ so that $f_{q k}^{(h)}=4 \sin ^{2}(\ell q) \sin ^{2}(\ell k)$, whereas the global bath is modeled by a collection of local baths of temperature $T_{g}=T$, each coupling to the occupation $\hat{a}_{i}^{\dagger} \hat{a}_{i}$ of one site with strength $\gamma / \sqrt{M}$, so that $R_{q k}^{(g)}=\sum_{i} R_{q k}^{(g i)}$ gives $f_{q k}^{(g)}=\sum_{i} 4 \sin ^{2}(i q) \sin ^{2}(i k) / M \simeq 1$.

In order to treat large systems and as a starting point for analytical approximations, we employ the meanfield approximation $\left\langle\hat{n}_{q} \hat{n}_{k}\right\rangle \approx\left\langle\hat{n}_{q}\right\rangle\left\langle\hat{n}_{k}\right\rangle$. It gives rise to a closed set of nonlinear kinetic equations for the mean occupations $\left\langle\hat{n}_{k}\right\rangle$ from which we obtain the steady state,

$$
\frac{\mathrm{d}\left\langle\hat{n}_{k}\right\rangle}{\mathrm{d} t}=\sum_{q}\left[A_{k q}\left\langle\hat{n}_{q}\right\rangle\left\langle\hat{n}_{k}\right\rangle+R_{k q}\left\langle\hat{n}_{q}\right\rangle-R_{q k}\left\langle\hat{n}_{k}\right\rangle\right]=0 .
$$

In the supplemental material we compare meanfield with exact Monte-Carlo results for $M=50$ and find excellent agreement [36] (see also Ref. [23] for a detailed description of both methods). Note that for a fixed ratio $\gamma_{h} / \gamma$, the steady state does not depend on $\gamma$. After having defined the system, we are now in the position to compute the steady-state mean occupations $\left\langle\hat{n}_{k}\right\rangle$ from Eq. (4).

Finite-size equilibrium condensation.-Let us first recapitulate the equilibrium case, where the system is coupled to a single bath of temperature $T$ only. Here one recovers the familiar grand-canonical mean occupations $\left\langle\hat{n}_{k}\right\rangle_{\mathrm{eq}}=\left[e^{\left(\varepsilon_{k}-\mu\right) / T}-1\right]^{-1}$, where the chemical potential $\mu$ has to be adjusted so that $\sum_{q}\left\langle\hat{n}_{k}\right\rangle_{\text {eq }}=N$. In the thermodynamic limit, $M \rightarrow \infty$ at constant density $n=N / M$, thermal fluctuations prevent the formation of a Bose condensate in a 1D system at finite temperature. However, for a finite system size $M$, a crossover into a Bose condensed regime with a relative occupation $\left\langle\hat{n}_{k_{c}}\right\rangle / N$ of order one in the ground state $k_{c}=\pi /(M+1)$ occurs when $T$ reaches the condensation temperature $T_{c}^{\mathrm{eq}} \approx 8.3 \mathrm{~nJ} / \mathrm{M}$, which we define as the temperature at which half of the particles occupy the single-particle ground states [36].

High-temperature nonequilibrium condensation.Turning to the nonequilibrium situation with both the global bath and the hot needle present, we have to compute the steady state by solving Eq. (4) numerically. Figure 1(b) shows the condensate fraction $N_{c} / N$, with occupation $N_{c}$ of the most populated mode $k_{c}$, versus both temperatures $T$ and $T_{h}$ for a system of 500 sites with $n=3, \ell=5$, and $\gamma_{h} / \gamma=0.5$. For small needle temperatures, $T_{h} \lesssim T$, we find a crossover into a Bose-condensed regime, roughly when the global temperature $T$ falls below the equilibrium value $T_{c}^{\text {eq }}$ (blue-white dotted line). However, when the needle temperature is increased further, an astonishing effect occurs: The global temperature at which condensation occurs increases by almost two orders of magnitude until it reaches a saturation value. Thus, for an environment well above the equilibrium condensation temperature $T_{c}^{\mathrm{eq}}$, coupling the system to a second, even hotter local bath (the hot needle) can induce Bose condensation. When the needle temperature is increased even further, we can observe another intriguing effect: the condensate is suddenly formed in an excited state, $k_{c} \approx \pi / \ell$, as indicated by the color code. Only for very large needle temperatures, condensation eventually breaks down completely.

In Fig. 1(c) the condensate fraction is plotted versus global temperature $T$ and system size $M$. One sees that 


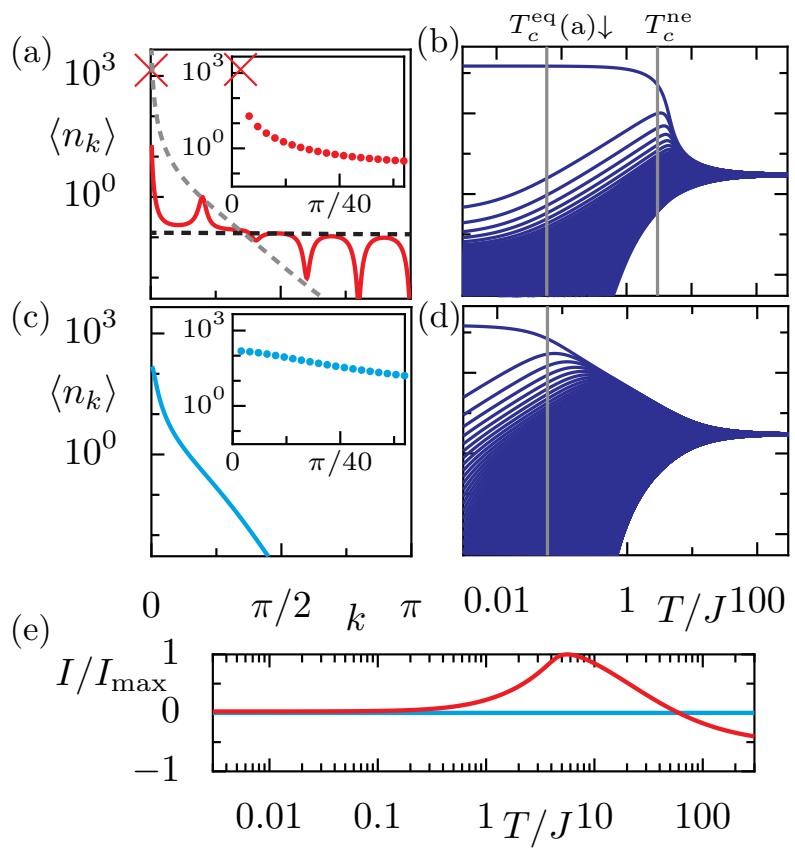

FIG. 2. (color online) (a,b) Mean occupations $\left\langle n_{k}\right\rangle$ for the parameters of Fig. 1 with $T_{h}=60 J$ and $M=500$, (a) versus $k$ [for $T=0.29 \mathrm{~J}$, black dot in Fig. 1(b)] and (b) versus $T$. In (a) the crosses indicate the condensate occupation, the gray (black) dashed line in the main panel shows thermal distributions for the temperature $T\left(T_{h}\right)$; the inset shows $\left\langle\hat{n}_{k}\right\rangle$ for small $k$. (c,d) Like (a,b), but for the equilibrium situation with $\gamma_{h} / \gamma=0$. Occupations obey the Bose-Einstein distribution. (e) Heat current $I$ from the needle through the system into the global bath versus $T$ for the nonequilibrium steady state [red line, parameters like in (b)], the blue line shows the trivial equilibrium value $I=0$.

up to large system sizes of about $10^{3}$ sites, condensation occurs at a large condensation temperature $T_{c}^{\text {ne }}$ that is practically independent of the system size. This behavior is reminiscent of the physics of Bose condensation in a three-dimensional (3D) system. Only at even larger system sizes, the condensation temperature decreases with $M$ resembling the equilibrium behavior in 1D. In the limit of small $M(\lesssim 100)$, again excited-state condensation in the mode $k_{c} \approx \pi / \ell$ is found.

Momentum distribution.-In order to obtain a better understanding of the intriguing behavior observed in Figs. 1(b) and (c), let us have a look at the full momentum distribution $\left\langle\hat{n}_{k}\right\rangle$. It is plotted in Fig. 2(a) for the parameters indicated by the black dot in Fig. 1(b). The occupation of the condensate formed in the ground state is indicated by a red cross and the occupation of all other modes by a red line. We find an unconventional nonmonotonous behavior of $\left\langle\hat{n}_{k}\right\rangle$ with equidistant peaks or dips. They are located around those wave numbers $\kappa_{\alpha}=\pi \alpha / \ell$ with $\alpha=0,1, \ldots, \ell$ that decouple from the hot needle, $f_{q \kappa_{\alpha}}^{(h)}=f_{\kappa_{\alpha} q}^{(h)}=0$. For momenta $k \approx \kappa_{\alpha}$ the distribution $\left\langle\hat{n}_{k}\right\rangle$ approximately follows a thermal dis- tribution with temperature $T$ (dashed gray line). Between the momenta $\kappa_{\alpha}$, the distribution roughly follows the thermal distribution associated with the hot temperature $T_{h}$ of the needle (dashed black line), which is rather flat. This behavior can be explained by noting that for $\left|\Delta_{q k}\right| \ll T_{b}$ the rates (2) become proportional to the bath temperature, so that the occupations $\left\langle\hat{n}_{k}\right\rangle$ are dominated by the hot bath with $T_{h} \gg T$, except for momenta near $\kappa_{\alpha}$ that almost decouple from the needle.

This discussion gives us already an idea of the mechanism behind the high-temperature condensation induced by the needle. Namely, the width of the peak of $\left\langle\hat{n}_{k}\right\rangle$ at $k=0$ is now determined by the competition between the global bath and the hot needle. The estimate $w=\left(\gamma / \ell \gamma_{h}\right) \sqrt{T / T_{h}}$ for the peak width can be obtained from requiring that $R_{q k}^{(h)} / R_{q k}^{(g)} \approx \gamma_{h}^{2} T_{h} f_{q k}^{(h)} /\left(\gamma^{2} T\right) \lesssim 1$ for $k<w$ and all $q$. It can be small compared to the width of the thermal distribution at temperature $T$ [Fig. 2(c)]. In this way long-wavelength excitations, which destroy Bose condensation in $1 \mathrm{D}$ equilibrium systems for temperatures above $T_{c}^{\mathrm{eq}}$, are reduced. The effect of Bose condensation and the dramatic increase of the condensation temperature $T_{c}$ induced by the hot needle can clearly be observed in Figs. 2(b) and (d), showing the $T$ dependence of the occupations $\left\langle\hat{n}_{k}\right\rangle$ for a system with and without coupling to the needle, respectively.

Estimating the condensation temperature.-Based on our qualitative discussion, we can estimate the nonequilibrium condensation temperature $T_{c}^{\text {ne }}$. In the condensate regime, where a large fraction $N_{c} / N$ of the particles occupy the ground-state mode $k_{0}=\pi /(M+1)$, the occupation of excited modes $k \ll 1$ in the vicinity of $k_{0}$ is approximately given by $\left\langle\hat{n}_{k}\right\rangle \approx N_{c} w^{2} /\left(2 M k^{2}\right)$ [36]. We obtain this expression from Eq. (4) as follows: We assume that all particles occupy long-wavelengths modes $k \ll 1$ (which is a valid approximation for $T \ll T_{c}^{\text {ne2 }}$, see next paragraph) and neglect the coupling of the hot bath to the condensate mode $k_{0}$, but not to other longwavelength modes. The condensation temperature, defined by $N_{c}=N / 2$, can then be estimated to read $T_{c}^{\text {ne1 }} \approx 30.6 \frac{\ell^{2} \gamma_{h}^{2} T_{h}}{\gamma^{2}} \frac{1}{M}$. It is plotted as red dotted line in Figs. 1(b) and (c) and agrees well with the observed behavior. Like in equilibrium in $1 \mathrm{D}$, also the nonequilibrium condensation relies on the infrared cutoff given by the inverse system size.

However, in contrast to equilibrium, in the nonequilibrium steady state Bose condensation can also be destroyed by increasing the occupation of modes with large momenta $q$, which couple to the condensate via the rates induced by the global bath. This effect can be estimated by considering the regime $T \ll T_{c}^{\text {ne1 }}$, where the total occupation of excited long-wavelength modes is suppressed by the finite system size $M$, so that we can approximate the occupation of excited states by the flat distribution $\left\langle\hat{n}_{q}\right\rangle \approx\left(N-N_{c}\right) / M$ induced by the dominant hot-needle 

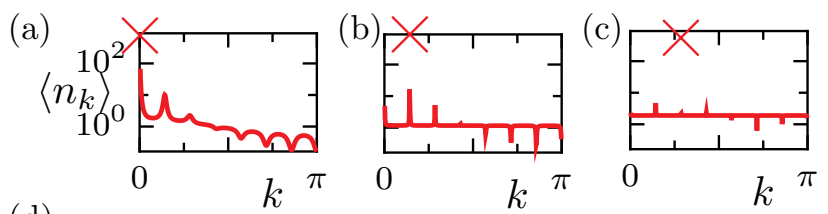

(d)

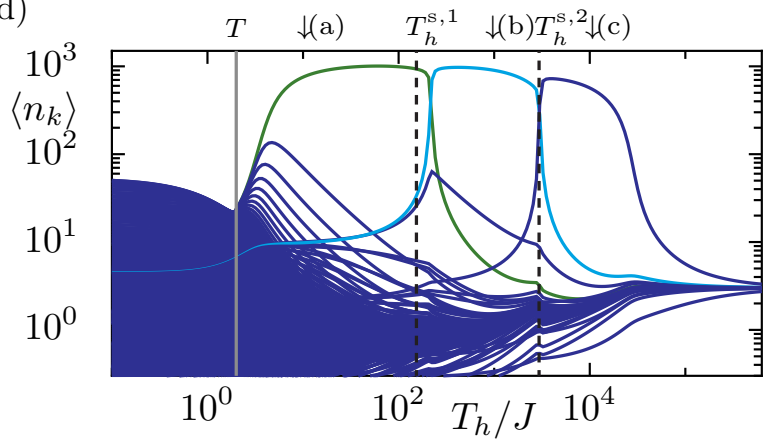

FIG. 3. (color online) Mode occupations (d) versus needle temperature $T_{h}$ for $T=2 J, \ell=7, M=500, n=3$, and $\gamma_{h} / \gamma=1$. With increasing $T_{h}$ the system passes from a state without Bose condensate through a sequence of states with a condensate in the states $k_{0} \approx 0$ (green line), $k_{1} \approx \pi / \ell$ (light blue line), and $k_{2} \approx 2 \pi / \ell$ (violet line), before condensation breaks eventually down again. (a-c) Momentum distribution for states with condensates in three different modes.

bath [dashed black line in Fig. 2(a)]. Considering the coupling of the so-occupied excited states to the condensate via the global bath, we find $N-N_{c}=T M /(2 J)$ [36]. From requiring $N_{c}=N / 2$, one finds the condensation temperature $T_{c}^{\text {ne2 }} \approx n J$, which is plotted as red solid line in Fig. 1(b) and (c). The fact that it does not depend on the system size, reflects similarity to the break-down of Bose condensation in a $3 \mathrm{D}$ system in equilibrium, which is not driven by long-wavelength modes either.

Excited-state condensation.-Figure 3(d) shows how the occupations depend on the needle temperature $T_{h}$ for a system with $\gamma_{h} / \gamma=1, M=500, \ell=7$, and $T=2 J$. Since the global temperature $T$ lies well above the equilibrium condensation temperature $T_{c}^{\mathrm{eq}} \approx 0.05 \mathrm{~J}$, no Bose condensate is found at $T_{h}=T$, where the system is in equilibrium. However, when $T_{h}$ is increased, soon ground-state Bose condensation sets in. When the needle temperature is increased further, remarkably a Bose condensate in the excited mode $k \approx \pi / \ell$ supersedes the ground-state condensate at a switch temperature $T_{h}^{\mathrm{s}, 1}$. The condensate mode switches once more to $k \approx 2 \pi / \ell$ at $T_{h}^{\mathrm{s}, 2}$, before eventually at very large needle temperatures $T_{h} \gtrsim 10^{4} J$ condensation breaks down again. The panels (a), (b), and (c) depict the momentum distribution for the three different needle temperatures marked in (d) and clearly show condensation in three different modes.

We find that the switching of the condensate mode is triggered by the possibility to reduce the coupling between the condensate mode and the hot needle. Namely, typically the allowed wave numbers $k=\nu \pi /(M+1)$ that comply with the boundary conditions, do not assume those values $\kappa_{\alpha}$ that would perfectly decouple from the hot needle. We denote by $k_{\alpha}$ the allowed wavenumber that minimizes the distance $\delta_{\alpha}=\left|k-\kappa_{\alpha}\right|$, which quantifies the coupling, $f_{q k_{\alpha}}^{(h)} \simeq 4 \sin ^{2}(\ell q) \ell^{2} \delta_{\alpha}^{2}$. With increasing $T_{h}$ we generally find a sequence of condensate modes $k_{0}, k_{\alpha_{1}}, k_{\alpha_{2}} \ldots$, where $\alpha_{j+1}$ is the smallest value of $\alpha$ with $\alpha>\alpha_{j}$ and $\delta_{\alpha}<\delta_{\alpha_{j}}$. The sequence ends, when the coupling cannot be lowered anymore by a larger $\alpha$. Since one has $\delta_{0}=k_{0}$ for the ground state $k_{0}$ and fluctuating values $\delta_{\alpha} \leq \delta_{0} / 2$ (depending on $M$ and $\ell$ ) for $\alpha \geq 1$, one always finds at least one switch of the condensate mode and $\alpha_{1}=1$. While for the parameters of Fig. 1(b) the sequence ends already with $k_{1}$, it ends with $k_{2}$ for the parameters of Fig. 3 (for $\ell=21$ and $M=200$, we observe the sequence $\alpha=0,1,2,7$, not shown).

We can estimate the switch temperature $T_{h}^{\mathrm{s} \alpha}$, above which a condensate in mode $k_{\alpha}$ forms. The momentum distribution can formally be written like $\left\langle\hat{n}_{k}\right\rangle=$ $\sum_{q} R_{k q}\left\langle\hat{n}_{q}\right\rangle / B_{k}$ with $B_{k}=\sum_{q}\left[A_{q k}\left\langle\hat{n}_{q}\right\rangle+R_{q k}\right]$. Physical occupations $\left\langle\hat{n}_{k}\right\rangle \geq 0$ require $B_{k}>0$. Assuming a condensate with $\left\langle\hat{n}_{k_{\alpha}}\right\rangle \approx N$ particles in mode $k_{\alpha}$, the temperature $T_{h}$ above which $\left\langle\hat{n}_{k_{\alpha^{\prime}}}\right\rangle \geq 0$ for all $\alpha^{\prime}<\alpha$ provides a good estimate for the switch temperature, $T_{h}^{\mathrm{s} \alpha} \approx 0.5 n\left(\gamma / \ell \gamma_{h}\right)^{2} \max _{\alpha^{\prime}<\alpha}\left(\Delta_{k_{\alpha} k_{\alpha^{\prime}}} / \delta_{\alpha^{\prime}}^{2}\right)[36]$, see dashed lines in Fig. 3(d) and Fig. 1(b). The linear dependence of $T_{h}^{\mathrm{s} \alpha}$ on $n$ indicates that excited-state condensation is suppressed ( shifted to $T_{h}=\infty$ ) in the limit of high densities, where ground-state condensation is expected for a system coupled to two thermal baths (of positive temperature) $[22,23]$. While we have presented examples for $\ell \ll M$, with the needle placed near the edge of the sample, and assumed this limit also in our analytical estimates, simulations show that the enhancement of the condensation temperature equally occurs for any needle position. However, placing the needle away from the edge we typically observe excited-state condensation, as it is indicated also by the $\ell^{-2}$ behavior of the estimated shift temperature $T_{h}^{\mathrm{s} \alpha}$.

General picture and conclusion-An intuitive interpretation of the high-temperature and excited-state Bose condensation observed here is that the nonequilibrium condensation can also be viewed as a mechanism that suppresses the heat influx $I_{h}$ from the hot bath, with $I_{b}=\sum_{q k} \Delta_{q k} R_{q k}^{(b)}\left(\left\langle\hat{n}_{q}\right\rangle+1\right)\left\langle\hat{n}_{k}\right\rangle$. Namely, keeping the distribution $\left\langle\hat{n}_{k}\right\rangle$ fixed, $I_{h}$ would increase linearly with $T_{h}$ [according to Eq. (2)], while it still has to be balanced by the outflux $I_{g}$ into the colder global bath (since $I_{h}=-I_{g}$ for a steady state). This increase is prevented by forming of a condensate in a mode that almost decouples from the hot needle. This interpretation is supported by Fig. $2(\mathrm{e})$ showing that the heat current $I=I_{h}=-I_{g}$ through the system plotted versus $T$ shows a maximum near the condensation temperature and, thus, a negative differential heat conductivity in the condensed regime. 
This counterintuitive effect is explained by noting that the number of particles contributing to the heat transport is reduced by condensing into the ground state $k_{0}$, which hardly couples to the hot needle. The onset first of ground-state condensation and later also of excited-state condensation observed when the needle temperature $T_{h}$ is increased [Fig. 3(d)] can, therefore, be understood as a strategy of the system to minimize its coupling to the hot needle (and with that $I_{h}$ ) further and further. Note that these intriguing effects do not rely on the discrete nature of the tight-binding chain considered here and, therefore, occur equally in a continuous system [36].

The tendency to avoid a state with strong coupling to the environment bears resemblance to the quantum Zeno effect [37]. The underlying mechanism, that the coupling to a second very hot bath can lead to a depletion of some system modes and, thus, to an enhanced occupation (and quantum degeneracy) of the subsystem defined by all other modes (not necessarily including the ground state), should be rather universal. It suggests a general strategy for the robust preparation of quantum degenerate nonequilibrium states with unconventional properties and also in large-temperature environments by realizing a hot bath coupling to all modes but a few. In future work, it will be interesting to explore such possibilities in bosonic and fermionic quantum systems. This includes the investigation of interacting systems and the role of stronger system-bath coupling.

Acknowledgment.-We acknowledge discussion with Toni Ehmcke. This work was supported by the German Research Foundation DFG (FOR 2414). D. V. is grateful for support by the Studienstiftung des deutschen Volkes.

* Electronic address: schnell@pks.mpg.de

$\dagger$ Electronic address: eckardt@pks.mpg.de

[1] M. Rigol and A. Muramatsu, Phys. Rev. Lett. 93, 230404 (2004).

[2] L. Vidmar, J. P. Ronzheimer, M. Schreiber, S. Braun, S. S. Hodgman, S. Langer, F. Heidrich-Meisner, I. Bloch, and U. Schneider, Phys. Rev. Lett. 115, 175301 (2015).

[3] M. Mitrano, A. Cantaluppi, D. Nicoletti, S. Kaiser, A. Perucchi, S. Lupi, P. D. Pietro, D. Pontiroli, M. Ricco, S. R. Clark, D. Jaksch, and A. Cavalleri, Nature 530, 461 (2016).

[4] M. Heyl, A. Polkovnikov, and S. Kehrein, Phys. Rev. Lett. 110, 135704 (2013).

[5] P. Jurcevic, H. Shen, P. Hauke, C. Maier, T. Brydges, C. Hempel, B. P. Lanyon, M. Heyl, R. Blatt, and C. F. Roos, arXiv:1612.06902 (2016).

[6] N. Fläschner, D. Vogel, M. Tarnowski, B. S. Rem, D.-S. Lümann, M. Heyl, J. C. Budich, L. Mathey, K. Sengstock, and C. Weitenberg, arXiv:1608.05616 (2016).

[7] D. M. Basko, I. L. Aleiner, and B. L. Altshuler, Ann. Phys. 321, 1126 (2006).

[8] R. Nandkishore and D. A. Huse, Ann. Rev. Cond. Mat. Phys. 6, 15 (2015).
[9] M. Schreiber, S. S. Hodgman, P. Bordia, H. P. Lschen, M. H. Fischer, R. Vosk, E. Altman, U. Schneider, and I. Bloch, Science 349, 842 (2015).

[10] J. Smith, A. Lee, P. Richerme, B. Neyenhuis, P. W. Hess, P. Hauke, M. Heyl, D. A. Huse, and C. Monroe, Nat. Phys. 12, 907 (2016).

[11] J.-y. Choi, S. Hild, J. Zeiher, P. Schaü̈, A. Rubio-Abadal, T. Yefsah, V. Khemani, D. A. Huse, I. Bloch, and C. Gross, Science 352, 1547 (2016).

[12] P. Ponte, Z. Papić, F. m. c. Huveneers, and D. A. Abanin, Phys. Rev. Lett. 114, 140401 (2015).

[13] A. Lazarides, A. Das, and R. Moessner, Phys. Rev. Lett. 115, 030402 (2015).

[14] V. Khemani, A. Lazarides, R. Moessner, and S. L. Sondhi, Phys. Rev. Lett. 116, 250401 (2016).

[15] C. W. von Keyserlingk, V. Khemani, and S. L. Sondhi, Phys. Rev. B 94, 085112 (2016).

[16] D. V. Else, B. Bauer, and C. Nayak, Phys. Rev. Lett. 117, 090402 (2016).

[17] J. Zhang, P. W. Hess, A. Kyprianidis, P. Becker, A. Lee, J. Smith, G. Pagano, I.-D. Potirniche, A. C. Potter, A. Vishwanath, N. Y. Yao, and C. Monroe, Nature 543, 217 (2017).

[18] S. Choi, J. Choi, R. Landig, G. Kucsko, H. Zhou, J. Isoya, F. Jelezko, S. Onoda, H. Sumiya, V. Khemani, C. von Keyserlingk, N. Y. Yao, E. Demler, and M. D. Lukin, Nature 543, 221 (2017).

[19] M. Bukov, L. D'Alessio, and A. Polkovnikov, Adv. in Phys. 64, 139 (2015).

[20] A. Eckardt, Rev. Mod. Phys. 89, 011004 (2017).

[21] N. Tsuji, T. Oka, and H. Aoki, Phys. Rev. Lett. 103, 047403 (2009).

[22] D. Vorberg, W. Wustmann, R. Ketzmerick, and A. Eckardt, Phys. Rev. Lett. 111, 240405 (2013).

[23] D. Vorberg, W. Wustmann, H. Schomerus, R. Ketzmerick, and A. Eckardt, Phys. Rev. E 92, 062119 (2015).

[24] L. E. F. Foa Torres, P. M. Perez-Piskunow, C. A. Balseiro, and G. Usaj, Phys. Rev. Lett. 113, 266801 (2014).

[25] K. I. Seetharam, C.-E. Bardyn, N. H. Lindner, M. S. Rudner, and G. Refael, Phys. Rev. X 5, 041050 (2015).

[26] H. Dehghani, T. Oka, and A. Mitra, Phys. Rev. B 91, 155422 (2015).

[27] G. Goldstein, C. Aron, and C. Chamon, Phys. Rev. B 92, 174418 (2015).

[28] T. Iadecola, T. Neupert, and C. Chamon, Phys. Rev. B 91, 235133 (2015).

[29] T. Shirai, J. Thingna, T. Mori, S. Denisov, P. Hänggi, and S. Miyashita, New J. Phys. 18, 053008 (2016).

[30] T. Qin and W. Hofstetter, arXiv:1704.03250 (2017).

[31] I. Carusotto and C. Ciuti, Rev. Mod. Phys. 85, 299 (2013).

[32] J. Klaers, J. Schmitt, F. Vewinger, and M. Weitz, Nature 468, 545 (2010).

[33] T. Byrnes, N. Y. Kim, and Y. Yamamoto, Nature Phys. 10, 803 (2014).

[34] H. A. M. Leymann, D. Vorberg, T. Lettau, C. Hopfmann, C. Schneider, M. Kamp, S. Höfling, R. Ketzmerick, J. Wiersig, S. Reitzenstein, and A. Eckardt, arXiv:1612.04312 (2017).

[35] H. Breuer and F. Petruccione, The Theory of Open Quantum Systems (Oxford University Press, Oxford \& New York, 2002).

[36] Additional information regarding equilibrium condensation, a comparison to exact Monte-Carlo simulations, the 
derivation of the condensation and the switch temperatures, as well as the physics of a continuous $1 \mathrm{D}$ system can be found in the supplemental material.
[37] B. Misra and E. C. G. Sudarshan, J. Math. Phys. 18, 756 (1977). 


\title{
Supplemental material for "High-temperature nonequilibrium Bose condensation induced by a hot needle"
}

\author{
Alexander Schnell, ${ }^{1, *}$ Daniel Vorberg, ${ }^{1}$ Roland Ketzmerick, ${ }^{1,2}$ and André Eckardt ${ }^{1, \dagger}$ \\ ${ }^{1}$ Max-Planck-Institut für Physik komplexer Systeme, Nöthnitzer Straße 38, 01187 Dresden, Germany \\ ${ }^{2}$ Technische Universität Dresden, Institut für Theoretische Physik and Center for Dynamics, 01062 Dresden, Germany
}

(Dated: July 28, 2017)

In this supplemental material we (i) estimate the condensation temperature for a one-dimensional tightbinding chain of finite length $M$ in equilibrium; (ii) justify the kinetic equations of motion obtained from the meanfield approximation by comparing it to Monte-Carlo results for the exact master equation; (iii) estimate the nonequilibrium condensation temperatures for the $1 \mathrm{D}$ and the 3D-like transition; (iv) estimate the switch temperatures for excited state condensation; and (v) show that the nonequilibrium condensation phenomena found for a discrete tight-binding chain can also be observed in a continuous one-dimensional system.

\section{CONDENSATION TEMPERATURE IN EQUILIBRIUM}

Under equilibrium conditions, when the system is coupled only to the global bath of temperature $T$ (i.e. for $\gamma_{h}=0$ ), Equation (4) (of the main text) is solved by the grand-canonical mean occupations

$$
\left\langle\hat{n}_{k}\right\rangle=\frac{1}{e^{\left(\varepsilon_{k}-\mu\right) / T}-1}
$$

with chemical potential $\mu$. When (finite-size) Bose condensation sets in, $\mu$ approaches $\varepsilon_{k_{0}}$ from below, so that the occupations of the low-energy modes with $k \ll 1$ can be approximated by

$$
\left\langle\hat{n}_{k}\right\rangle \simeq \frac{T}{\varepsilon_{k}-\mu} \simeq \frac{T}{J k^{2}-2 J-\mu},
$$

where we have used $\varepsilon_{k}=-2 J \cos (k) \simeq-2 J+J k^{2}$. The chemical potential can be expressed in terms of the occupation $N_{c}=\left\langle\hat{n}_{k_{0}}\right\rangle$ of the ground state with wave number $k_{0}=\pi /(M+1)$,

$$
\mu=-2 J+J k_{0}^{2}-T / N_{c} .
$$

For low temperatures, the number $N^{\prime}$ of particles occupying excited states, with $k=\nu \pi /(M+1)$, is dominated by the long-wavelength modes, so that we can approximate

$$
N^{\prime}=\sum_{k^{\prime} \neq k_{0}}\left\langle\hat{n}_{k}\right\rangle \simeq \sum_{\nu=2}^{\infty} \frac{1}{\frac{J \pi^{2}}{T M^{2}}\left(\nu^{2}-1\right)+\frac{1}{N_{c}}} .
$$

For a finite system, we define the characteristic temperature $T_{c}$, where Bose condensation sets in, as the tem-

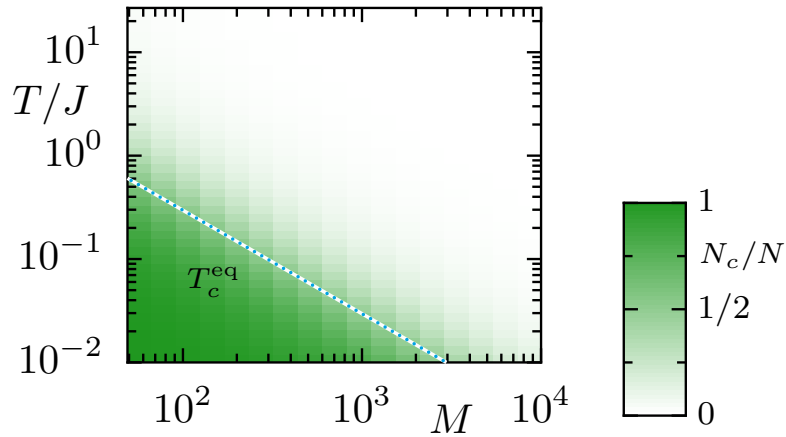

FIG. 1. Condensate fraction $N_{c} / N$ for a tight binding chain of $M$ sites at temperature $T$ (shading). The blue-white dotted line gives the analytical estimate for the condensation temperature, where half of the particles occupy the single-particle ground state.

perature for which half of the particles occupy the singleparticle ground state, $N^{\prime}=N_{c}=N / 2$. It is given by

$$
T_{c}^{\mathrm{eq}} \simeq \frac{a \pi^{2}}{2} \frac{n J}{M} \approx 8.3 \frac{n J}{M},
$$

where $a \approx 1.68$ solves $1=a \sum_{\nu=2}^{\infty} 1 /\left(\nu^{2}+a-1\right)$. In Fig. 1 we plot the ground-state occupation (i.e. the condensate fraction) of the tight binding chain together with the estimate (A.5) for the condensation temperature $T_{c}^{\mathrm{eq}}$. The inverse dependence of $T_{c}^{\mathrm{eq}}$ on the system size $M$ reflects the well-known result that in one spatial dimension, in the thermodynamic limit Bose-Einstein condensation is suppressed by thermal long-wavelength fluctuations.

\section{QUASIEXACT MONTE-CARLO RESULTS FOR A SMALL SYSTEM}

The meanfield approximation $\left\langle\hat{n}_{q} \hat{n}_{k}\right\rangle \approx\left\langle\hat{n}_{q}\right\rangle\left\langle\hat{n}_{k}\right\rangle$, which gives rise to the closed set of kinetic equations (4) for the mean occupations, allows us to treat large systems of up to $M=10^{4}$ lattice sites and to find analytical estimates for the parameters where condensation sets in. In order to justify this approximation, we have also simulated the full many-body rate equation for the probability distribution $p_{\boldsymbol{n}}$ for finding the system in the eigenstate $|\boldsymbol{n}\rangle$. It reads

$$
\dot{p}_{\boldsymbol{n}}=\sum_{k q}\left(1+n_{q}\right) n_{k}\left(R_{k q} p_{\boldsymbol{n}_{q \leftarrow k}}-R_{q k} p_{\boldsymbol{n}}\right),
$$




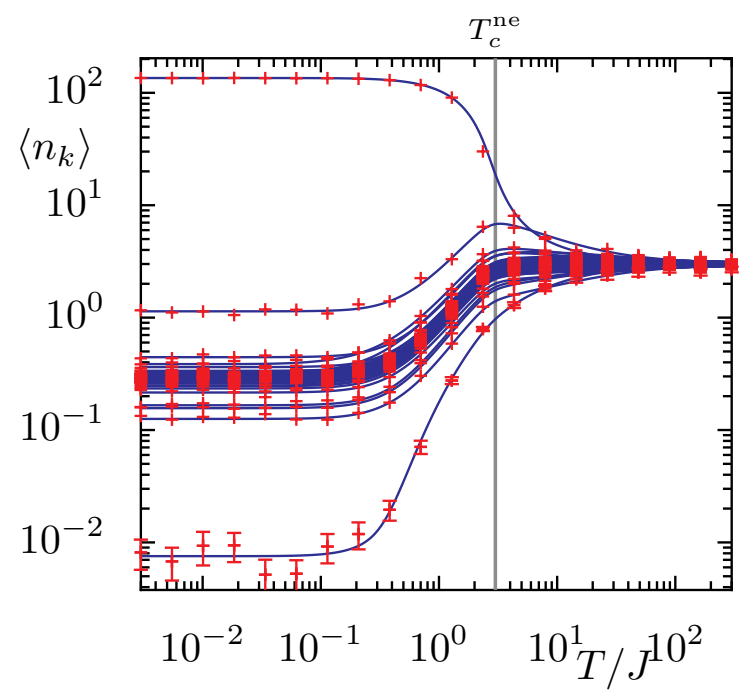

FIG. 2. Mean occupations $\left\langle n_{k}\right\rangle$ for a system with $M=$ $50, n=3, l=3, \gamma=\gamma_{h}, T_{h}=120 \mathrm{~J}$. Crosses represent the Monte-Carlo results. We show error bars for the least occupied state only, because for the other states they are too small to be visible. The data is well approximated by the solid lines which result from the meanfield approximation.

where $\boldsymbol{n}_{q \leftarrow k}$ denotes the vector of occupation numbers obtained from $\boldsymbol{n}$ by transferring a particle from mode $k$ to mode $q$.

An efficient way of solving this equation is given by quantum-jump Monte-Carlo simulations (see, e.g., [1]). For that purpose we generate a random walk in the classical space of Fock states $\boldsymbol{n}$ (which is exponentially large with respect to the system size, but much smaller than the Fock space, which contains also the coherent superpositions of the Fock states). Namely, according to the sum and the relative weight of the many-body rates $R_{q k}\left(n_{q}+1\right) n_{k}$ leading away from the current state $\boldsymbol{n}$, we draw both the time after which a quantum jump happens and the new state $\boldsymbol{n}_{q \leftarrow k}$, respectively. Expectation values like the mean occupations $\left\langle\hat{n}_{k}\right\rangle$ are computed by averaging over a random path. This method gives quasiexact results, in the sense that the accuracy is controlled by the length of the random path. A detailed description of the method is given in Ref. [2].

In Fig. 2 we plot the mean occupations $\left\langle\hat{n}_{k}\right\rangle$ of a system of $M=50$ sites and $n=3, \ell=3, \gamma_{h} / \gamma=1$, and $T_{h}=120 \mathrm{~J}$. The Monte-Carlo data (red crosses) are reproduced almost perfectly by the meanfield solution (solid lines). Already in this rather small system, we can see a rather sharp crossover, to a Bose condensed regime at $T_{c}^{\text {ne }}$.

\section{NONEQUILIBRIUM CONDENSATION TEMPERATURE}

As described in the main text, in our system a Bose condensate in the ground state $k_{0}=\pi /(M+1)$ can be destroyed either by a large occupation of long-wavelength modes or by a large occupations of excited states at all energies. While the former case resembles condensation in a 1D system in equilibrium and leads to a systemsize dependent condensation temperature $T_{c}^{\text {ne1 }}$, the latter case bears similarity to equilibrium condensation in 3D and happens at a condensation temperature $T_{c}^{\text {ne2}}$, which is independent of the system size. Below, we will estimate both temperatures. The generalization to excited-state condensation is straightforward and not presented here.

\section{Condensation destroyed by long-wavelength modes}

Let us suppose that the system is in the condensed regime, where a large fraction $N_{c} / N$ of the particles occupy the ground-state mode and the modes at large quasimomenta are approximately unoccupied (which requires $T \ll T_{c}^{\text {ne2}}$, see next section). The mean occupations $\left\langle\hat{n}_{k}\right\rangle$ of the long-wavelength modes $k$ with $k_{0}<k \ll 1$ in the vicinity of $k_{0}$ are then obtained from

$$
\begin{aligned}
0 \approx A_{k k_{0}}^{(g)}\left\langle\hat{n}_{k}\right\rangle N_{c}+R_{k k_{0}}^{(g)} N_{c}+ \\
\sum_{q \neq k_{0}} A_{k q}\left\langle\hat{n}_{k}\right\rangle\left\langle\hat{n}_{q}\right\rangle+\sum_{q \neq k_{0}} R_{k q}\left\langle\hat{n}_{q}\right\rangle-\sum_{q} R_{q k}\left\langle\hat{n}_{k}\right\rangle,
\end{aligned}
$$

which was derived from Eq. (4) by neglecting the weak residual coupling of the ground-state mode to the hot needle, $R_{k k_{0}}^{(h)} \approx 0 \approx A_{k k_{0}}^{(h)}$. The last term describes the coupling to approximately empty modes at larger quasimomenta $q$.

We can now use the assumption of a large needle temperature $T_{h} \gg T$. Employing the scaling $\left\langle\hat{n}_{k}\right\rangle \propto T / T_{h}$ (which will be shown to be self-consistent below), we find

$$
\begin{aligned}
0= & R_{k k_{0}}^{(g)} N_{c}+\sum_{q \neq k_{0}} R_{k q}^{(h)}\left\langle\hat{n}_{q}\right\rangle-\sum_{q} R_{q k}^{(h)}\left\langle\hat{n}_{k}\right\rangle \\
& +\mathcal{O}\left(T / T_{h}\right),
\end{aligned}
$$

where we obtained the scaling of $R_{k q}$ and $A_{k q}$ from Eqs. (2) and (3) from the main text. The rates $R_{k q}^{(h)}$ scale like $k^{2}$ for small $k$. So for $k$ going to zero, we may omit the second term, but not the third one since $\left\langle\hat{n}_{k}\right\rangle$ is peaked around zero, which will keep the third term finite. This yields

$$
\begin{aligned}
0 & \approx R_{k k_{0}}^{(g)} N_{c}-\sum_{q} R_{q k}^{(h)}\left\langle\hat{n}_{k}\right\rangle \\
& \approx \gamma^{2} T N_{c}-2 \gamma_{h}^{2} \ell^{2} k^{2} M T_{h}\left\langle n_{k}\right\rangle .
\end{aligned}
$$

For the second approximation we used the small- $\left|\Delta_{k q}\right| / T$ expression, Eq. (2), for the rates $R_{k k_{0}}^{(g)}$ and $\sum_{q} R_{q k}^{(h)} \approx$ 
$4 \gamma_{h}^{2} T_{h} \sin ^{2}(\ell k) \sum_{q} \sin ^{2}(\ell q) \approx 2 \gamma_{h}^{2} \ell^{2} k^{2} M T_{h}$. Thus, for large $T_{h}$ we have

$$
\left\langle\hat{n}_{k}\right\rangle=\frac{N_{c} \gamma^{2} T}{M 2 \gamma_{h}^{2} \ell^{2} T_{h} k^{2}}=\frac{N_{c} w^{2}}{2 M} \frac{1}{k^{2}},
$$

with $w$ as defined in the main text. The occupations scale like $T / T_{h}$ as required by self consistence. Even though expression (A.11) is derived for modes $k$ with $k_{0}<k \ll 1$, we can use it for all $k \neq k_{0}$ since it vanishes rapidly with increasing $k$. With that, the condensate depletion due to the long wave modes $N^{\prime}=\sum_{k \neq k_{0}}\left\langle\hat{n}_{k}\right\rangle$ is approximately given by

$$
N^{\prime} \approx N_{c} w^{2} \frac{M}{2 \pi^{2}} \sum_{\nu=2}^{\infty} \frac{1}{\nu^{2}} .
$$

Using the definition that at the condensation temperature $N_{c}=N^{\prime}=N / 2$, we can estimate

$$
T_{c}^{\mathrm{ne} 1} \approx 30.6 \frac{\ell^{2} \gamma_{h}^{2} T_{h}}{\gamma^{2}} \frac{1}{M} .
$$

\section{Condensation destroyed by modes with large quasimomentum}

Let us again suppose that the system is in the condensed regime with a large fraction $N_{c} / N$ occupying the ground-state mode. But now, we assume that $T \ll T_{c}^{\text {ne1 }}$ so that the long-wavelengths modes are hardly occupied. Furthermore, we assume that the occupations of the most of the excited modes $k \neq k_{0}$ are close to a flat distribution corresponding to the hot temperature $T_{h} \gg J$, so that we can approximate $\left\langle\hat{n}_{k}\right\rangle \approx N^{\prime} / M$ for $k \neq k_{0}$. Plugging this ansatz into the rate equation (4) we find for $k=k_{0}$

$$
0 \approx \frac{N^{\prime}}{M} N_{c} \sum_{q} A_{k_{0} q}^{(g)}+\frac{N^{\prime}}{M} \sum_{q \neq k_{0}} R_{k_{0} q}^{(g)}-N_{c} \sum_{q \neq k_{0}} R_{q k_{0}}^{(g)} .
$$

Analogous to Eq. (A.7), here we assumed that the ground state $k_{0}$ decouples from the needle. In the condensed regime we find $N_{c} \gg T / J$, and $N_{c} \gg N^{\prime} / M$ and therefore the first and the last term dominate over the second one. Using $\sum_{q} A_{k_{0} q}^{(g)}=\gamma^{2} \sum_{q} \Delta_{q k_{0}} \approx \gamma^{2} 2 J M$ this leads to

$$
0 \approx \gamma^{2} N_{c}\left[2 J N^{\prime}-T s(T)\right]
$$

where we have defined the sum

$$
s(T)=\sum_{q \neq k_{0}} \frac{\Delta_{q k_{0}} / T}{e^{\Delta_{q k_{0}} / T}-1} \stackrel{T \gg J}{\longrightarrow} M-1 .
$$

So we find the total depletion to be given by

$$
N^{\prime}=\frac{T s(T)}{2 J} .
$$

Solving this equation for $N_{c}=N^{\prime}=n M / 2$, we may obtain $T_{c}^{\text {ne2 }}$ numerically. However, with the limiting value of $s\left(T_{c}^{\mathrm{ne} 2}\right) \approx M-1$ from Eq. (A.16) the condensation temperature reads $T_{c}^{\text {ne2 }} \approx n J$, which is consistent with the assumption $T_{c}^{\mathrm{ne} 2} \gg J$ as long as $n \gg 1$.

\section{TEMPERATURE FOR SWITCH TO EXCITED STATE CONDENSATION}

Let us now discuss, under which conditions we can expect condensation in an excited state, $k_{c}=k_{\alpha} \approx$ $\frac{\pi}{\ell}, \frac{2 \pi}{\ell}, \ldots$, in the finite system. The mean field equation (4) must hold for all $k$, which we may solve formally like

$$
\left\langle n_{k}\right\rangle=\frac{\sum_{q} R_{k q}\left\langle n_{q}\right\rangle}{\sum_{q}\left(A_{q k}\left\langle n_{q}\right\rangle+R_{q k}\right)} .
$$

We will use the fact that all occupations must be nonnegative, $\left\langle n_{k}\right\rangle \geq 0$, to find the regime where excited state condensation may occur.

Since the numerator of Eq. (A.18) is strictly positive, the denominator has to be positive, too. Deep in the condensed regime, we may neglect the depletion, $N_{c} \approx$ $N$, which yields

$$
A_{k_{c} k} N+\sum_{q} R_{q k}>0 .
$$

Considering that the condensate decouples from the hot bath, the rate imballance $A_{k_{c} k}$ is dominated by the global bath and we can approximate $A_{k_{c} k} \approx \gamma^{2} \Delta_{k k_{c}}$. Additionally $T_{h} \gg T$ holds, such that $\sum_{q} R_{q k} \approx \sum_{q} R_{q k}^{(h)} \approx$ $\gamma_{h}^{2} T_{h} 2 M \sin (k \ell)^{2}$. In total, we have to satisfy

$$
\gamma^{2} \Delta_{k k_{c}} N+\gamma_{h}^{2} T_{h} 2 M \sin (k \ell)^{2}>0
$$

for all $k$. For ground-state condensation $k_{c}=k_{0}=$ $\pi /(M+1)$ both terms are positive, such that for all parameter values the ground state condensate ansatz does not violate the physical requirement of positive occupations. For $k_{\alpha} \approx \kappa_{\alpha}=\frac{\alpha \pi}{\ell}$ with $\alpha \geq 1$ however, the first term is negative for all $k<k_{\alpha}$ and must be compensated by the second term. Since the second term is minimal at one of the decoupling wavenumbers $k_{\alpha^{\prime}}, \alpha^{\prime}<\alpha$, it suffices to check for positivity at these wave numbers. From this requirement, we find that for

$$
T_{h}>T_{h}^{s, \alpha}=\frac{n \gamma^{2}}{2\left(\ell \gamma_{h}\right)^{2}} \max _{\alpha^{\prime}<\alpha}\left(\frac{\Delta_{k_{\alpha} k_{\alpha^{\prime}}}}{\delta_{\alpha^{\prime}}^{2}}\right)
$$

condensation in the excited state $k_{\alpha}$ may occur.

\section{CONTINUOUS SYSTEM}

In the main text, we treated a one-dimensional (1D) system of noninteracting bosons in a tight-binding lattice. However, the effects discussed in the main text do 


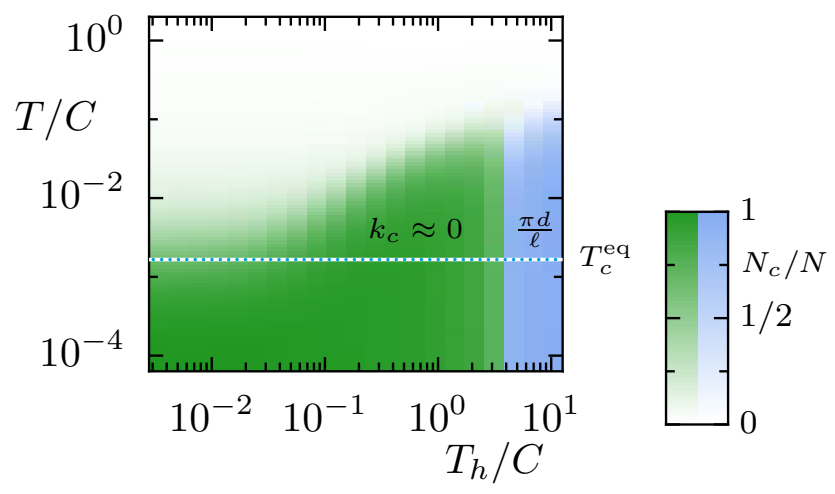

FIG. 3. Condensate fraction $N_{c} / N$ for the continuous $1 \mathrm{D}$ system of length $L=500 d$ and density of $n=0.1 / d$ versus the temperatures $T$ and $T_{h}$ of the global bath and the hot needle, respectively. The spatial extent of the hot needle is $d$ and it is placed at distance $\ell=20 d$ from the edge. The relative coupling between both baths is $\gamma_{h} / \gamma=1 / 4$. Green (light blue) shading indicates the relative number of particles in the mode $k_{0} \approx 0\left(k_{1} \approx \pi d / \ell\right)$. The blue-white dotted line corresponds to the condensation temperature in equilibrium.

not depend on the discrete nature of the tight-binding lattice. They occur in a similar form also in a continuous 1D system described by the Hamiltonian

$$
\hat{H}=\int_{0}^{L} \mathrm{~d} x \hat{\psi}^{\dagger}(x)\left(-\frac{\hbar^{2}}{2 m} \frac{\mathrm{d}^{2}}{\mathrm{~d} x^{2}}\right) \hat{\psi}(x)=\sum_{k} \varepsilon_{k} \hat{n}_{k},
$$

where $m$ denotes the mass of the particles and $\hat{\psi}(x)$ the field operator annihilating a boson at position $x$. The dimensionless wavenumbers $k=\nu \pi d / L$ with $\nu=1,2, \ldots$ and some length scale $d$ (which we take to be the extent of the hot needle defined below) characterize the single-particle eigenstates with energy $\varepsilon_{k}=\frac{\hbar^{2} k^{2}}{2 m d^{2}} \equiv C k^{2}$, and wave functions $\langle x \mid k\rangle=\sqrt{2 / L} \sin (k x / d)$. The corresponding number operator reads $\hat{n}_{k}=\hat{c}_{k}^{\dagger} \hat{c}_{k}$ with $\hat{c}_{k}=$ $\int_{0}^{L} \mathrm{~d} x\langle k \mid x\rangle \psi \hat{(x)}$. The energy eigenstates of the system are Fock states $|\boldsymbol{n}\rangle$ labeled by the vector of occupation numbers $n_{k}$.

In such a continuous system, a hot local bath at position $x=\ell$ of spatial extent $d$, can be described by the coupling operator

$$
\hat{v}_{h}=\frac{L}{d} \int_{\ell-\frac{d}{2}}^{\ell+\frac{d}{2}} \mathrm{~d} x \hat{\psi}^{\dagger}(x) \hat{\psi}(x) .
$$

The corresponding single-particle rates are of the form of Eq. (2), with $f_{k q}^{(h)}=\left[\cos \left(k \frac{\ell}{d}\right) \operatorname{sinc}(k / 2)-\right.$ $\left.\cos \left(q \frac{\ell}{d}\right) \operatorname{sinc}(q / 2)\right]^{2}$ giving $f_{k q}^{(h)} \simeq 4 \sin ^{2}(k \ell / d) \sin ^{2}(q \ell / d)$ in the limit $d \rightarrow 0$. A global heat bath is still described by Eq. (2)with $f_{k q}^{(g)}=1$.

From these rates we computed the corresponding mean-occupations for a system of $N=0.1 \mathrm{~L} / \mathrm{d}$ particles, length $L=500 d$, and needle position $\ell=20 d$. In Fig. 3 we plot the condensate fraction, i.e. the fraction of particles occupying the most occupied mode whose wave number is indicated by the color of the shading (green for $k_{0} \approx 0$ and light blue for $k_{1} \approx \pi d / \ell$ ). As for the tight-binding chain, we can clearly see that the temperature of the global bath at which the system condenses increases with needle temperature. As a result Bose condensation is found for a system coupled to two baths both having temperatures well above the equilibrium condensation temperature $T_{c}^{\text {ne }}$ (which is indicated as blue-white dotted line). Moreover, when the needle temperature is increased further, ground-state condensation in mode $k_{0} \approx 0$ is superseded by the formation of a condensate in the excited mode $k_{1} \approx \pi d / \ell$, which provides a better decoupling from the hot needle.

* Electronic address: schnell@pks.mpg.de

$\dagger$ Electronic address: eckardt@pks.mpg.de

[1] M. B. Plenio and P. L. Knight, Rev. Mod. Phys. 70, 101 (1998).

[2] D. Vorberg, W. Wustmann, H. Schomerus, R. Ketzmerick, and A. Eckardt, Phys. Rev. E 92, 062119 (2015). 